\title{
Stewart-Treves Syndrome of the Lower Extremity*
}

\author{
Rossana Ruth Garcia da Veiga ${ }^{1}$ \\ Alessandra Haber Carvalho ${ }^{1}$ \\ Maraya de Jesus Semblano Bittencourt ${ }^{1}$
}

\author{
Bianca Angelina Macêdo do Nascimento ${ }^{1}$ \\ Arival Cardoso de Brito ${ }^{1}$
}

DOI: http:/ / dx.doi.org/10.1590/abd1806-4841.20153926

\begin{abstract}
A bstract: Stewart-Treves syndrome is a rare cutaneous angiosarcoma that develops in long-standing chronic lymphedema. Though most commonly this angiosarcoma is a result of post mastectomy lymphoedema, it also develops in Milroy disease, idiopathic, congenital, traumatic and filarial lymphoedema. Despite the rarity of this syndrome and its poor prognosis, early diagnosis associated with radical surgery can provide improved survival. We report a case of angiosarcoma in the lower limb in a patient with chronic lymphedema associated with history of repeated erysipela episodes.
\end{abstract}

Keywords: Lymphedema; Neoplasms, vascular tissue; Sarcoma

\section{INTRODUCTION}

With the evolution of cutaneous oncology and dermatologic surgery, dermatologists have been increasingly called upon for the diagnosis and therapeutic management of rare tumors. ${ }^{1}$ The development of angiosarcoma in chronic lymphedema was first described by Stewart and Treves in 1948 in women with breast cancer treated with radical mastectomy. Literature also report cases of the syndrome in the lower extremities, in congenital, idiopathic, traumatic, infectious or post-surgical lymphedema. ${ }^{2,3}$ Early recognition of suspicious lesions in a lymphomatous limb and biopsy are essential, due to the poor prognosis of this entity, with a high rate of local recurrence, systemic dissemination and poor survival rate in five years. ${ }^{1,4}$

\section{CASE REPORT}

Female patient, 46 years old, with a history of bilateral lymphedema evolving for 28 years. Five months ago she noticed the appearance in the right leg of a bleeding tumor, with foul-smelling secretion drainage. Patient had previous history of deep venous thrombosis and recurrent erysipelas. The dermatological examination showed exuberant lymphedema in both legs (Figure 1A). Right leg presented multiple erythematous nodules on brown sclerotic area and 4 angiomatous tumors of exulcerated surface (Figure 1B). Anatomopathological examination of the tumor showed neoplastic proliferation of nodular architecture composed of epithelioid cells with pleomorphic vesicular nuclei, prominent nucleoli and abundant eosinophilic cytoplasm. Also

Received on 10.08.2014.

Approved by the Advisory Board and accepted for publication on 11.09.2014.

Study performed at Dermatologia da Universidade Federal do Pará (UFPA) - Belém (PA), Brazil.

Financial Support: None.

Conflict of Interest: None.

1 Universidade Federal do Pará (UFPA) - Belém (PA), Brazil.

(C)2015 by Anais Brasileiros de Dermatologia 

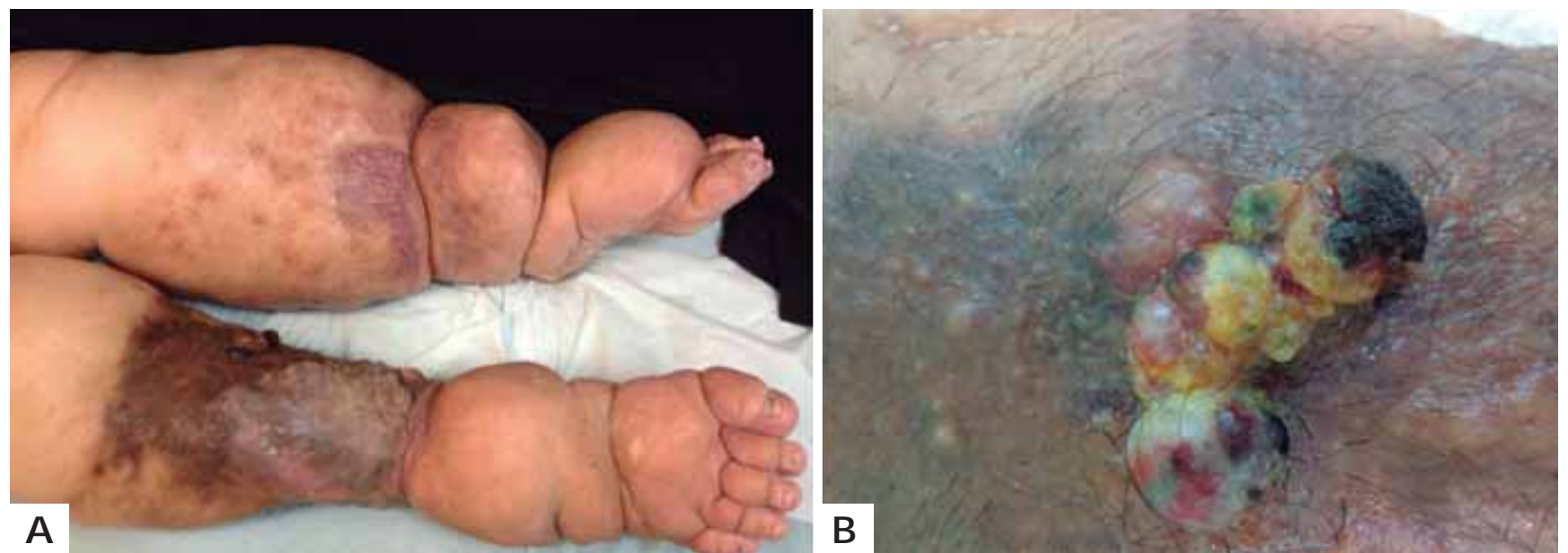

Figure 1: A: Exuberant lymphedema in both legs. Right leg presents multiple tumors on brown sclerotic plaque. B: Detail of the tumor area, site of the biopsy

pronounced vascular proliferation represented by large vascular channels lined by atypical endothelial, some with thrombus and hemorrhagic and necrotic areas (Figure 2). Immunohistochemical study revealed a diffuse expression of CD31 and CD34, confirming the diagnosis of epithelioid angiosarcoma in chronic lymphedema area, featuring Stewart-Treves syndrome. CT was requested to screen distant metastasis and the patient was sent to a referral hospital in Oncology in the State.

\section{DISCUSSION}

Stewart-Treves syndrome (STS) is a rare syndrome associated with chronic lymphedema, classically described in the arm after radical mastectomy followed by radiation therapy. In $10 \%$ of cases it occurs in other locations, ${ }^{2,3}$ as in this case report, in which an unusual presentation in the lower limb was noted. STS represents $5 \%$ of cases of angiosarcomas, ${ }^{5}$ with about 400 cases reported in the literature. It occurs most often between the fifth and seventh decade of life.

Angiosarcomas are malignancies that originate from cells with endothelial function and morphology of blood or lymphatic vessels. In the presence of lymphoedema, they grow as plaques or cutaneous and subcutaneous nodules, single or multiple, which may coalesce, with an unknown etiology. Because it is a poorly differentiated neoplasms, it requires confirmation by immunohistochemistry., ${ }^{1,3}$ The disease demonstrates positivity for endothelial cell markers such as factor VIII and anti-CD34 and antiCD31 antibodies, the latter being the one with greater sensitivity and specificity. ${ }^{2,45}$ The infrequent occurrence of this disease and the innocuous appearance of the tumor lead to delays in diagnosis and treatment. ${ }^{3}$
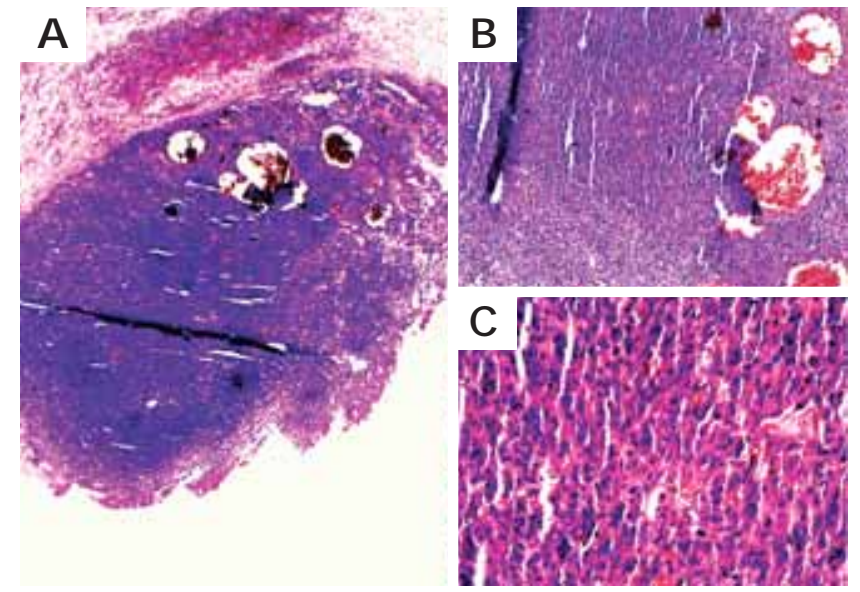

FIgURE 2: A: Dermal neoplastic proliferation of nodular architecture, consisting of epithelioid cells with pleomorphic vesicular nuclei, prominent nucleoli and abundant eosinophilic cytoplasm (HE, x4). B: Highlight of the associated pronounced vascular proliferation, represented by large vascular channels lined by atypical endothelial, some with thrombi and areas of hemorrhage and necrosis (HE, x10). C: Atypical epithelioid cells in detail (HE, x40)

Surgeries indicated for patients with malignant vascular tumors vary according to the possibility of preserving the member, and when preservation is not possible, the indicated procedure is the amputation, but radiotherapy and chemotherapy are also recommended. Angiosarcoma is a highly malignant tumor, both in its visceral location as in soft tissue, being the malignant vascular tumor with the worst prognosis. $^{2,5}$ Patient survival is 19-31 months from 
diagnosis, with a high rate of local recurrence $(84 \%$ in 5 years) and distant metastases, especially pulmonary, being the main cause of death. Five-year survival rates are between $10 \%$ and $35 \% .^{2,3,4,5}$ In conclusion, the

\section{REFERENCES}

1. Fleury LFFJ, Sanches JAJ. Primary cutaneous sarcomas. An Bras Dermatol. 2006;81:207-21.

2. McHaffie DR, Kozak KR, Warner TF, Cho CS, Heiner JP, Attia S. Stewart-Treves Syndrome of the Lower Extremity. J Clin Oncol. 2010;28:e351-2.

3. Chopra S, Ors F, Bergin D. MRI of angiosarcoma associated with chronic lymphoedema: Stewart-Treves syndrome. Br J Radiol. 2007;80:e310-3.

4. Silva ER, Cavalcante EF, Ferreira FVA, Soares LRC, Juaçaba SF, Ximenes RA. Síndrome de Stewart Treves: relato de caso. Rev Bras Ginecol Obstet. 2003;25:211-4.

5. Lee JH, Jeong YJ, Oh DY, Kim SW, Rhie JW, Ahn ST. Clinical experience of stewarttreves syndrome in the lower leg. Arch Plast Surg. 2013;40:275-7. authors reported a case of STS in the lower limb. We must maintain a high index of suspicion in skin lesions in chronically swollen member, even those of benign appearance, because early treatment can improve the potential for patient survival. \begin{tabular}{l}
\hline M AILIN G ADDRESS: \\
M araya de Jesus Semblano Bittencourt \\
91, Generalíssimo D eodoro A venue, \\
U marizal \\
66055-240 Belém, PA. \\
Phone: (91) 3201-6894. \\
Email:marayabittencourt@hotmail.com
\end{tabular}

H ow to cite this article: Veiga RRG, Nascimento BAM, Carvalho AH, Brito AC, Bittencourt MJS. Stewart-Treves Syndrome of the Lower Extremity. An Bras Dermatol. 2015;90(3 Supl 1):S232-4. 